International Journal of Child, Youth and Family Studies (2013) 3.1: 475-488

\title{
DILEMMAS OF PRACTICE IN THE ECOLOGY OF EMANCIPATORY YOUTH-ADULT PARTNERSHIPS
}

\author{
Jeanette Roach, Esayas Wureta, and Laurie Ross
}

\begin{abstract}
This article explores dilemmas that arise when using a participatory, experiential neighborhood problem-solving and planning program in settings that have different expectations and beliefs about youth and adults partnering in organizational and community decision-making. Using Bronfenbrenner's (1979) ecology of human development and Wong, Zimmerman, and Parker's (2010) pyramid of youth participation, a series of dilemmas are explored. These dilemmas include: negotiating challenges of power; scaling up youth-adult partnerships into organizational decisionmaking and governance; reconciling tensions between practices, principles, and values when disseminating a program from one organization to another; dealing with organizational events that occur outside the youth program; and succumbing to pressure to achieve funder-derived outcomes. Two insights emerge from the analysis of these dilemmas. First, young people embrace adult-provided structure when adults and young people are not ready to work in emancipatory youth-adult partnerships. Second, as we move toward emancipatory youth-adult partnerships, the developmental sphere of youth programs has to expand to include the activities, relationships, and roles that traditionally have been limited to organizational leadership and governance. Likewise the developmental sphere of the governing body has to incorporate the activities, relationships, and roles of what has typically been the youth program.
\end{abstract}

Keywords: youth-adult partnerships, ecological analysis, organizational readiness

Jeanette Roach is a graduate student in the Department of International Development, Community, and Environment at Clark University, 950 Main Street, Worcester, Massachusetts, USA 01610. E-mail: jroach@clarku.edu

Esayas Wureta is a Program and Outreach Specialist at Peace Corps in Washington, DC, USA. E-mail: ewureta@peacecorps.gov

Laurie Ross, Ph.D. (the corresponding author) is an Associate Professor in the Department of International Development, Community, and Environment at Clark University, 950 Main Street, Worcester, Massachusetts, USA 01610. E-mail: lross@clarku.edu 
Youth-adult partnerships are multi-dimensional with many sets of competing forces to manage (Ross, Downs, Tejani, Dezan, \& Lowe, 2010). Youth characteristics, adult characteristics, organizational features, and societal norms and dynamics can facilitate and/or constrain shared decision-making and democratic processes between youth and adults. An ecological framework is useful to analyze the ways identities, values, beliefs, practices, roles, and relationships intersect within and across multiple settings in the context of power differences (Bronfenbrenner, 1979).

In this article we apply an ecological analysis to explain dilemmas and challenges we faced in our attempt to forge authentic youth-adult partnerships during the Urban Community Action Planning with Teens (UCAPT) program. UCAPT is a participatory, experiential program designed to engage young people, who are affected by oppressive conditions in their communities, in a neighborhood problem-solving and planning process in partnership with adults (Ross, 2002; Ross \& Coleman, 2000). We have used the UCAPT program with groups of young people in an economically distressed northeastern city in the United States called Union ${ }^{1}$.

During our time facilitating UCAPT, we have been both participants and observers ${ }^{2}$. Occupying the dual role of youth worker and researcher, we have had unique insider and outsider status, in which we have gone through continuous cycles of action and reflection. Once the programs were completed, we spent time reflecting on the extent to which we were able to forge authentic partnerships with the youth as well as to achieve community change outcomes. The two case studies presented below are the result of this retrospective reflection.

\section{Applying an Ecological Framework to UCAPT Case Studies}

An ecological framework is based on the idea that human development occurs in an environmental context consisting of a set of nested structures (Bronfenbrenner, 1979). The first structure is the microsystem - or the child's immediate development contexts, such as the family, the school, the peer group, and neighborhood. The basic infrastructure of a microsystem consists of the activities, relationships, and roles within that context. The second is the mesosystem - or the relationships among microsystems (e.g., the nature, structure, and level of congruence in the relationships between the family and the school or between a neighborhood and a peer group). The third is the exosystem, defined as the contexts in which the child is not immediately present, but which directly influence the microsystem (e.g., parents' employment situation; school board

\footnotetext{
${ }^{1}$ All names of people (aside from the authors), organizations, and locations have been changed to protect the identity of individuals and the context in which this work took place.

${ }^{2}$ The "we" in this case refers to Esayas, an Eritrean graduate student in his mid-20s who was hired to run UCAP-A at the Union Youth Center; Jeanette, a graduate student and staff member of the Union Youth Center in her early 20s who grew up in Union; and Laurie, a professor at a local university who developed the Urban Community Action Planning for Teens (UCAPT) curriculum.
} 
International Journal of Child, Youth and Family Studies (2013) 3.1: 475-488

policies). The fourth is the macrosystem, defined as the consistencies in the form and content of micro, meso, and exosystems at the level of the subculture or the culture as a whole.

Case One involves Esayas's work at the Union Youth Center. Three dilemmas emerged in this case. Two of the dilemmas revolve around Esayas's challenges to relinquish his power while simultaneously convincing the young people that they had power in the UCAPT program. The third dilemma consists of the struggles he observed the youth facing when they attempted to exercise their new disposition and skills in working in partnership with adults in the larger organizational context. The ecological framework helps to explain how there can be different understandings, expectations, and practices in the extent to which youth and adults engage in shared decision-making in the context of one organization.

Case Two involves Jeanette's work at the Oak Hill Neighborhood Center. This case poses a set of dilemmas about what happens when an organization actively seeks to adopt the UCAPT curriculum in their youth development programs without having a mission and practices that are fully aligned with youth-adult partnership. In Case Two, the ecological framework allows us to examine microsystem and exosystem factors that explain why an organization would be attracted to UCAPT but face significant challenges implementing the program with fidelity to its underlying values and principles.

\section{Case One: Negotiating Power and Scaling Up Youth Participation in the Union Youth Center}

During summer 1991, a large group of youth gathered in front of the Union Court House to pass the idle summer days. Their presence was unwelcomed by the larger public, however, and the young people were arrested and charged with loitering. As a result of this highly publicized incident, a group of youth and adults started to meet to tackle issues ranging from the lack of youth spaces to police harassment and unemployment. They called themselves the Young Adult Action Group (YAAG).

After several years of meetings and research, this group of young people and several adults opened the Union Youth Center. The center was to be free of charge to any young person in the city and to be a safe space where everyone agreed gang affiliations and turf issues would be left at the door. Most importantly, they retained the YAAG as the organizational structure that ensured youth would participate in operating the organization, shaping programs, and delivering activities, and that youth would also be voting members of the board of directors (Ross, 2002). For many years, young people literally ran the center. However, as the organization grew and operations became more complex, the governance and leadership roles young people played diminished. The YAAG became more of a youth leadership program than an organizational decision-making body.

Today, the Union Youth Center serves hundreds of Union youth and young adults as an educational and social facility that provides activities such as martial arts, a recording studio, a dance studio, recreational activities, and structured education, leadership, and workforce development programs. While the center remains committed to youth voice, their input is more limited to the realm of programming and planning activities rather than governance. UCAP-A, 
International Journal of Child, Youth and Family Studies (2013) 3.1: 475-488

one of the structured programs and the focus of this case study, is a youth leadership program that infuses the arts as a medium for social change within the framework of Urban Community Action Planning with Teens (UCAPT).

\section{Urban Community Action Planning - Arts (UCAP-A): Program Design and Implementation}

Esayas: The organization's executive director conceptualized and designed UCAP-A based on her love for the arts, her connections with the arts community, and the organization's commitment to using the UCAPT curriculum in its structured programs. She realized that many of the youth in the center possessed strong arts skills, but for various reasons were not receiving the necessary training, and lacked opportunities to utilize their passion and skills for personal and community development. She believed that the arts could be a powerful motivating force to engage young people in social change efforts in their communities. The director partnered with the Union Art Museum to create such an opportunity. UCAP-A incorporated social change and the arts with elements of work readiness and experiential learning for the purpose of fostering leadership skills and developing more protective measures in young people’s lives.

We held the program twice a week. One of the weekly sessions focused on engaging the youth in community analysis. The other weekly session was spent at the Union Art Museum, where the youth learned about various art media. The 15 youth participants, made up of males and females ranging from 15 to 20 years of age, included a mix of long-time Union Youth Center members, as well as students of area public high schools who were recruited for this program.

I conveyed to the group right from the beginning that UCAP-A would be led by them, and that they would be engaging in community analysis. I made it clear through my words and a variety of team builders and icebreakers that the youth would be making important decisions. I often told them, "This is a program for you and by you, and so you have to take the initiative."

On days that were focused on community analysis, I facilitated discussions, community walks, mapping exercises, and consensus-building activities in order to identify an issue that the group would address collectively. While engaging in community walks, we observed distinct differences between neighborhoods where the UCAP-A participants lived and those "on the other side of the city.” The housing stock in the neighborhoods where the UCAP-A participants lived was inundated with trash, litter, and illegal dumping. The young people talked about how others saw their neighborhoods as full of gang activity, as bad, and as unsafe. While they recognized that the issues were complex, they chose trash and littering as the subject for an intervention because they felt more likely to have an impact on that issue.

After reaching consensus on trash, Jeanette and I helped the youth conduct research to understand the trash collection system in Union. During this research phase, the young people were also receiving art lessons at the Union Art Museum. At first, they spent time in the museum learning various art media and interacting with artists. Their arts education would soon take a practical direction as they developed ideas for projects.

They decided to address trash and littering by creating a video that taught younger youth the environmental and community effects of littering, and holding an art exhibit that expressed 
International Journal of Child, Youth and Family Studies (2013) 3.1: 475-488

their outrage about the social and environmental effects of the abundance of litter in their community. We initiated a third project to increase the number of trash receptacles in the community, but after working on this initiative for a month the youth chose to focus on the other projects given the costs, time, and complexity associated with the endeavor.

The culmination of the program was the completion of a short video project and an art exhibition at the center held in conjunction with artists and staff from the Union Art Museum. Sixty members of the community came out to support the work of the young people, including the funder, Union Youth Center staff, board members, youth members, and their families. UCAP-A program participants reported a sense of pride in their artwork. The youth were eager to display the products of their work, and discuss the UCAP-A process with attendees.

The UCAP-A program was a success to the extent that it allowed youth the space to think critically and address issues that affect their lives and community. The emancipatory and experiential learning aspects of the program contributed to the many positive outcomes. Below are some outcomes achieved by youth involved in the program:

- $\quad 70 \%$ of youth partners increased by a letter grade in one subject or more.

- $100 \%$ of youth partners who were seniors in high school enrolled in college (7/15).

- $100 \%$ of youth partners who were out of school and unemployed obtained employment after the program $(2 / 15)$.

- $100 \%$ of youth partners who were involved in the juvenile justice system did not reoffend $(3 / 15)$.

In addition to these individual-level outcomes, the group produced sculptures that depicted their perceptions of causes and consequences of trash and inequities in the trash collection system in the city. They raised their own as well as the community's awareness of the problem and demonstrated that youth are concerned and want to address trash and littering.

\section{Dilemma \#1: Getting Youth to Believe they have Power}

While the above description of UCAP-A process and outcomes appears to be relatively smooth, we now discuss challenges we faced in the program. An initial barrier we encountered was youth's mindset about their lack of decision-making power vis-à-vis adults. The youth-adult partnership aspect of the program intrigued many of the youth, yet, they were dubious about the "power" they were told they now possessed. As one participant, Cara, stated, “Adults say you have power, but they never mean it. When it comes to actually using your power, they are quick to take your 'power' away.”

To address this tension, we provided opportunities that would build group camaraderie and egalitarian social relations. We started each meeting with an icebreaker that focused on team building and experience sharing, and we supplemented the formal meetings with field trips and potlucks. We realized we had to permit ourselves to be present with the youth and not always focused on moving to the next step of the program. Being present allowed us to hear youth concerns and ideas, and helped foster an atmosphere of mutual respect. Being present allowed us to joke and play with youth and, at times, to be the subject of their humor. 
Even with these intentional activities, youth were quick to revert to typical youth roles and expectations, that is, to follow adult instruction and not behave as partners. For example, in the weeks leading up to the group's decision to discontinue the trash receptacle project, youth questioned why we let them pursue the project if it was not realistic. They had expected that we would make the decision for them and stop the project.

\section{Dilemma \#2: Getting the Adults to Relinquish Decision-Making Power}

Allowing young people to make decisions, even if we as adults do not think they are the right decisions, is a critical component of youth-adult partnerships. This became evident during UCAP-A while hiring museum artists to partner with the youth on their projects. Initially, Jeanette and I selected an artist we thought worked very well with youth and possessed a high caliber of art skills. We made this decision without the approval of the youth. When we informed the group, the youth put the brakes on the process. They sat us down and stated their preference for another artist. Although we believed the artist we had chosen had the experience to better deliver program outcomes, we honored the youth preference because it was more valuable than program outcomes such as high quality artwork. In the end, we worked well with the youth's preferred artist, and more importantly, it allowed the youth to engage in real decision-making.

We realized that at the heart of our challenge to relinquish decision-making power was our concern about fulfilling the programmatic outcomes expected by the funder. Our move to be open and honest with the youth about funder mandates opened a whole new space for dialogue about how one works within boundaries set by outside actors. We were able to explain why we made the decision we did about the artist. Rather than thwarting youth voice or hiding information from the youth, we found that being clear and open about funder requirements actually proved to be liberating in that the youth had the information they needed to make good decisions about the direction of their project.

By the end of program, Cara, the same youth quoted earlier, stated that the program, "left me feeling very confident in my abilities. It pushed me to create something that was solely me. In reflecting, I feel like because of this program I can do anything.” Youth were able to form authentic relationships with adult partners within the space of the program. The processes involved in UCAP-A allowed for an organic engagement that dispelled traditional youth adult relationships and empowered both adult and youth partners in becoming active agents of change in their communities.

\section{Dilemma \#3: Scaling up Youth-Adult Partnerships}

The UCAP-A program created a space where youth and adult partners could equitably engage in community change. Unfortunately, the feelings of emancipation and power that were exercised in the UCAP-A program faced contestation in the greater Union Youth Center. Marla's experience exemplifies this challenge. She had been a regular participant for several years and had participated in almost all the programs available prior to her involvement in UCAP-A. Marla easily embraced the relinquishing of "adult power" and accepted the responsibilities that came with taking leadership. Once the program was over, however, Marla had a difficult time 
International Journal of Child, Youth and Family Studies (2013) 3.1: 475-488

navigating the structures of the center. Having grown accustomed to taking leadership and maneuvering the UCAP-A program, her relationship with the director and other staff members became almost confrontational.

The deterioration of Marla's relationship with adults in the organization was caused by the difference between youth and adult interactions in the center as a whole compared to the UCAP-A program. Although the center embraced youth-adult partnerships in the context of one program, other programs operating in the center did not follow such a philosophy. Moreover, the organization's structure, staff, and management did not fully internalize youth-adult partnership and thus failed to create an organizational culture that catered to such a philosophy. For example, while the organization's by-laws call for a certain number of youth to sit on the board of directors, it had been years since a consistent group of youth participated on this governing body. Further, the YAAG - which at one time had been the heart of youth-adult decision making at the organization level - had largely been defunct for at least a year prior to the UCAP-A group starting.

As a result of the UCAP-A program, youth reported a sense of empowerment and ability to affect change in their communities. Without supportive systems at the organization level, however, youth-adult partnerships realized in UCAP-A did not scale up to equitable relationships among youth and adults in the center as a whole. Although the center has some structures for youth inclusion, this has not translated to an overall culture or practice where youth are truly partners in the operation of the organization.

\section{Case Two: Reconciling Tensions between Practices, Principles, and Values in the Context of Organizational Events and Pressure to Achieve Measurable Outcomes at the Oak Hill Neighborhood Center}

Jeanette: Oak Hill Neighborhood Center is a neighborhood-based, multi-service community center providing low-income children and families in Union with a full spectrum of programs and services. Individuals and families often turn to Oak Hill Neighborhood Center for assistance in meeting their critical basic needs, to obtain shelter and transitional housing, and for after-school and summer recreational programs for young people.

Historically, the organization's youth programming has functioned as an alternative to the street and focused solely on providing sports and recreational opportunities. More recently, the organization has undergone a grant-funded restructuring process, and created the Child, Youth and Family Services Department. Within this restructuring, a principal goal emerged to shift Oak Hill Neighborhood Center toward the delivery of high quality youth development, leadership, and education programs for teens and young adults ages 14 to 18. As the Child, Youth and Family Services Department took shape, the director of the department engaged in conversations with the Executive Director of the Union Youth Center regarding the possibilities for organizational collaboration. Together, they identified UCAPT as the first programmatic step for the newly created department, and they envisioned it as the youth-led planning model for the development of an Oak Hill Neighborhood Center youth leadership program. 
International Journal of Child, Youth and Family Studies (2013) 3.1: 475-488

Management from Oak Hill Neighborhood Center and the Union Youth Center partnered to design the initial phase of UCAPT. I was identified as the Union Youth Center staff member who would support Oak Hill during the implementation of the pilot program due to my experience with Esayas facilitating UCAP-A. After the initial pilot, it was envisioned that Oak Hill Neighborhood Center staff would maintain the program.

As conceived by upper level leadership within Oak Hill Neighborhood Center, the program's primary objective of adopting UCAPT was the formation and promotion of an ongoing youth peer leadership team. The program was intended to allow young people to plan, shape, and lead future programming, and to provide a means through which to formalize the roles of young people as leaders within the organization. The pilot UCAPT program would provide the initial context for developing youth-adult partnerships essential for achieving this goal. During the early implementation stage, however, many organizational challenges emerged.

Beginning with recruitment and ending with the completion of a group project, issues around organizational and staff capacity issues emerged. The challenges included a disconnect between the principles underlying UCAPT and the actions of leadership and staff, communication barriers among stakeholders, inadequate time and resources, and particular events that transpired during implementation.

\section{UCAPT Implementation}

Prior to the first session with youth participants, I provided UCAPT training to two of the newly hired Oak Hill Neighborhood Center Teen Program staff members who were to be cofacilitators of the program. David, the Oak Hill recreation director, was responsible for youth recruitment. Unfortunately, he had not attended the UCAPT training, and potential participants were told that the program would consist of community service projects. They were not told that they would be working in partnership with staff to understand and address neighborhood issues through the design of a youth leadership program. The young people therefore came into the program with an erroneous understanding of program expectations and goals due to staff not fully understanding the principles underlying the program.

Despite challenges recruiting and retaining young people in the program, a core group of six youth, both male and female, met twice per week for 11 weeks. I led these sessions with the assistance of an Oak Hill Neighborhood Center staff member, Amanda. Having participated in UCAP-A, I was able to facilitate the same set of team builders and neighborhood analysis activities that had been used by Esayas. Through the UCAPT process, the group determined that youth gangs and disrespect for the neighborhood by residents were the main issues they wanted to tackle. By the eighth week of the program, the group had begun to realize that they had a role to play as youth leaders within Oak Hill Neighborhood Center. They discussed the importance of positive role models in ending the cycle of young people entering gangs; in particular, youth began to formulate ways they could be the role models for younger children attending Oak Hill Neighborhood Center. In this process, they highlighted how David had played an important role in preventing them and other youth in Oak Hill from joining gangs, and how he had engaged them in positive activities. 
The neighborhood analysis portion of the program discussed above progressed more slowly than it had for the UCAP-A group in the Union Youth Center. The incorrect program description used during recruitment contributed to the slow pace, and many of the young people expressed frustration with the format of the program. Originally told that the group would go out to various places in the community to perform service, the young people recruited were initially resistant to the idea that they would explore their neighborhood and select the issue(s) to address. As a result, I had to facilitate additional team-building activities and engage participants in more intensive discussions about neighborhood strengths. We took several field trips to community organizations, including the Union Youth Center, before they could begin to visualize their ability as youth to have an impact in the community. These factors, in addition to the staff turnover that postponed the program launch, pushed back the solution generation portion of the program.

With the pressure to have a tangible product completed by the end of the program, the Director of Child, Youth and Family Services handed a project to the group. The data collection and action planning portions of UCAPT were cut short, and the focus of the group turned from addressing gang issues to redesigning Oak Hill's teen space, a project that had recently received grant funding. Though the young people did not develop the project idea, they made it their own and carried it out by brainstorming ideas for how to transform the room, selecting paint and furniture, creating a scaled drawing of the new layout, and painting the space. Given their struggles during the problem identification and solution generation phases of UCAPT, and having articulated feelings of powerlessness in discussions around community issues, youth participants welcomed the clearly defined project.

Just as the task of painting the room was undertaken, the program ended. Oak Hill Neighborhood Center had begun its summer programming, and the staff would no longer be able to commit time to the UCAPT program. From start to finish, this pilot revealed some of the issues and dilemmas that can arise when an organization attempts to adopt innovative programs and practices without first internalizing the underlying principles and processes.

\section{Dilemma \#1: Reconciling Tensions between Practices, Principles, and Values}

Even though Oak Hill leadership desired organizational change, they seemed unready to adopt the key elements of UCAPT. Communication barriers and time constraints prevented stakeholders at all levels of the organization from coming to agreement about the purpose and principles of UCAPT both prior to and during implementation. Senior leadership was often consumed by broader organizational operations and did not have time to engage in reflective dialogue with staff and the youth. As a result, little shared understanding of UCAPT and youthadult partnerships emerged and staff had unclear expectations about their roles and responsibilities. This issue became evident during the recruitment process for UCAPT during which young people were given an inaccurate description of the program. It became even more evident during program implementation where staff never fully viewed young people as partners in UCAPT.

Amanda took a more traditional approach to youth work. Whenever a young person behaved inappropriately according to her expectations, such as being loud, using profanity, or 
joking around with others in the group, she would quickly reprimand them. Her reactive approach meant that she consistently asserted her authority rather than processing and redirecting negative behavior. This precluded relinquishing power; the staff member saw her role as maintaining order and attending to behavioral issues rather than engaging in a partnership with young people. For their part, youth participants were also accustomed to the traditional approach, and were more responsive to instruction than collaboration within the program.

\section{Dilemma \#2: Dealing with Organizational Events}

During the UCAPT pilot, it became clear that events within the organization but external to the program can have unanticipated impacts on processes and outcomes. At the height of the program, the morale of the youth and staff involved in UCAPT was dealt a serious blow with the firing of David. Though this staff person was not directly involved in UCAPT beyond recruitment, he was a central institutional figure for all of the young people involved. With his unexpected departure, many in the group shared sentiments that the Oak Hill Neighborhood Center leadership did not care about their wants and needs. As a result, they felt that their input and voices were not important, thereby undoing a great deal of the progress that had been made in the program. Though the group had begun articulating the leadership roles they could play in the organization - as role models for younger children, for example - this event undermined their fragile sense of power. Unaccustomed to incorporating young people's voices in organizational decision-making, Oak Hill leadership did not create the space for the youth leaders to share their grievances, let alone participate in the decision.

\section{Dilemma \#3: Succumbing to Pressure to Achieve Tangible Outcomes}

Through the processes of neighborhood problem and strength identification, young people partnering in the UCAPT program reached a point where they were beginning to articulate the role that the Oak Hill Neighborhood Center plays in their community. They were also beginning to identify the roles that young people can play in the community, and the contributions they could make to Oak Hill. Despite this achievement, the act of having a predetermined project handed to the group undermined the burgeoning youth-adult partnership and its underlying processes. During the eighth week of the program, the teen space redesign project was handed to them as the final task. With the program's end looming, we decided to take it on because of pressure to achieve a tangible outcome. Although the project was pushed onto them, youth exercised their agency by embracing the process to accomplish the task through the planning and design process. They seized the opportunity to design the teen space as they saw fit, from the choice of paint colors to the furniture and layout of the room.

In the end, the project undertaken by the group represents a missed opportunity for shifting the Oak Hill Neighborhood Center from providing services to young people to a space where they collaborate with adults. Rather than having a legitimate opportunity to impact the youth programming and policies of Oak Hill as intended, the project they were given channeled the group's energy into completing a one-time assignment. The project itself was very successful, and had the potential to be an important component of team-building and fostering youth-adult partnerships. What should have been a "small win" as the group of young leaders progressed during UCAPT unfortunately became the end in and of itself. 
International Journal of Child, Youth and Family Studies (2013) 3.1: 475-488

\section{Analysis of the Case Studies}

In the two case studies, youth and adults were confronted with a series of dilemmas when attempting to work in partnership. UCAPT - as a set of activities - was a constant in the Union Youth Center and the Oak Hill Neighborhood Center cases. Yet, the types of youth-adult relationships that emerged in the UCAP-A group differed from those within the Union Youth Center as an organization, and again from those that formed in the UCAPT program at the Oak Hill Neighborhood Center. If the program (UCAPT) in all three contexts was the same, and there was even some overlap in adult staff involved in the two cases, then what accounts for the different outcomes we saw in the types of youth participation and youth-adult partnerships that emerged in each case? In order to answer this question, we join Bronfenbrenner's (1979) ecology of human development and Wong, Zimmerman, and Parker's (2010) pyramid of youth participation into an ecological model of organizational readiness for youth-adult partnerships.

In Case One, it appears that youth and adults were able to achieve what Wong et al. call a pluralistic form of youth participation in the UCAP-A program:

Although youth-adult partnerships may have varying degrees of youth and adult control within them, shared planning and decision making is what differentiates the pluralistic type from other participation types.... The shared control between youth and adults provides a social arrangement that is ideal for positive youth development and empowerment. In this type, adults are involved at a level where the purpose of their presence is to maximize conditions and opportunities. (p. 109)

Within the UCAP-A microsystem, adults worked intentionally to create a space where young people understood the funder-driven parameters in which they could work and then ceded as much power as possible to them. This is not to say the adults stepped back and let the young people do whatever they wanted. To the contrary, adults made a conscious decision to "be" with the young people, to work alongside them. An excellent example of the youth's decision-making ability was when they expressed their dissatisfaction with the artist that was chosen for them by the adults. The adults heard them out and then, as a group, they hired the artist that the youth felt would be a better match.

An interesting clash between microsystems happened, however, when young people who had been through the UCAP-A program attempted to make decisions at the level of the organization. These young people found that while they were listened to, they did not play a role in setting the agenda at board meetings, or in creating organization-wide policy. This caused frustration for some of the youth. We see that while the organization espouses a youth-led model, in practice, young people's participation can be characterized as symbolic:

[Y]outh have the opportunity to voice their perspectives about problems and their potential solutions, and be heard by decision-makers. Adults may, for example set up formal or informal structures for youth to express their opinions and experiences...but in the end youth often do not have much power in the decisionmaking or agenda-setting process. (Wong et al., p. 108) 
International Journal of Child, Youth and Family Studies (2013) 3.1: 475-488

There appeared to be firm boundaries between the microsystem of the UCAP-A program and the exosystem of organizational leadership, including the board of directors. The center's history of youth-adult partnership and culture of deep youth involvement supported the pluralistic relationships within UCAP-A but did not guarantee such relationships at the level of organizational decision-making and governance.

Turning to the second case, we see that the Oak Hill Neighborhood Center actively sought to incorporate the UCAPT program as a foundational element of its newly revitalized youth development programming. Oak Hill leaders genuinely were interested in deepening youth involvement both in the organization and in the community. Yet, young people's participation was not fully realized. Youth involvement in the program could be described as what Wong et al. call vessel participation: "the main objective of youth presence is to advance an adult-driven agenda” (p. 106). Oak Hill Neighborhood Center appreciated the idea of youth involvement and wanted to develop a youth leadership program but the organization had not made a fundamental shift in culture that embraced or understood authentic youth participation. Staff continued to take the role of maintaining order and control in the group, rather than working in partnership with the young people.

Similar to the Union Youth Center, there was a firm barrier between the microsystem of the group and the exosystem where organizational decision-making and governance occurred. Decisions and activities in the exosystem (e.g., funding directives and hiring or firing decisions) negatively affected the extent to which young people could engage in authentic participation and decision-making in their program. In contrast to the Union Youth Center, where youth involvement - albeit a symbolic form - had become the norm, we found that the Oak Hill Neighborhood Center operated in a more traditional mode with adults having all of the decisionmaking power. Indeed, Oak Hill Neighborhood Center revealed some of the issues and dilemmas that can arise when an organization attempts to adopt innovative programs and practices without first internalizing the underlying principles and processes (Zeldin, Petrokubi, \& MacNeil, 2008).

\section{Discussion}

We have joined Bronfenbrenner's ecology of human development and Wong et al.'s pyramid of youth participation into an ecological model of organizational readiness for youthadult partnerships. We illustrated the importance of analyzing different ecological levels particularly the microsystem and exosystem - to understand the extent to which young people and adults forge pluralistic relationships and whether their efforts have emancipatory effects at the individual, group, organization, and community levels. The joining of these two frameworks into an ecological model of organizational readiness reveals both how different forms of youthadult partnerships can coexist within one organization, as well as the benefits and consequences of these various forms of youth participation. Analyzing dilemmas in this way provides a more nuanced understanding of the practice of working in youth-adult partnerships than much of the current literature provides.

Two interesting insights can be derived from analyzing these dilemmas through an ecological model of organizational readiness for youth-adult partnerships. First, the literature 
suggests that youth disengage when they are not allowed to have voice and decision-making power, particularly in a program that is geared toward promoting youth leadership and youthadult partnership (Zeldin, 2004). However, this is not what happened in the Oak Hill Neighborhood Center case, in spite of the vessel form of participation that young people exercised. In fact, they actually seemed to embrace adult-provided structure and projects. We found that neither the young people nor the adults were ready to work in a youth-adult partnership in which the adults cede power in order for the youth and adults to contribute and participate equitably and appropriately given their roles and status.

Second, and perhaps of deeper significance to our understanding of emancipatory youthadult partnerships, our cases suggest that to achieve pluralistic youth-adult partnerships the microsystem needs to expand to include what would typically be the exosystem in other settings. For example, there would not be a separate space where decisions about funding and staff are made. Rather, the functions of youth programs and governing boards would overlap. In the UCAP-A case, we did see the microsystem of the group expand to include exosystem-type decisions - such as the hiring of a particular artist and having the information needed to negotiate funder expectations. Interestingly, the Oak Hill case presented similar personnel challenges and the need to negotiate funder expectations; however, all decisions and information about these topics remained firmly in the exosystem, to the detriment of the formation of authentic youthadult partnerships.

These insights have implications for understanding organizational readiness and for guiding additional research into youth-adult partnerships. Staff and leaders in the organization from top to bottom need to fully consider the extent to which the principles that underlie authentic youth-adult partnerships are compatible with the setting in which they are being introduced. If there is not yet compatibility, then the organization needs to engage in deliberate dialogue until there is a sense of shared perceptions among actors regarding these core values, principles, and processes. Successful incorporation of youth-adult partnerships in organizational governance and decision-making also requires that the organization fully considers the time, energy, adult qualifications, and youth readiness it takes for pluralistic participation (Zeldin et al., 2008). Finally, the move toward emancipatory youth-adult partnerships involves the expansion of the developmental sphere of youth programs to include the activities, relationships, and roles that traditionally have been limited to organizational leadership and governance structures. Likewise, the developmental sphere of the governing body has to take on activities, relationships, and roles of what has typically been the youth program. Achieving this merging of youth program microsystems and governance exosystems will require new types of ecologically informed research and practice. 
International Journal of Child, Youth and Family Studies (2013) 3.1: 475-488

\section{References}

Bronfenbrenner, U. (1979). The ecology of human development: Experiments by nature and design. Cambridge, MA: Harvard University Press.

Ross, L. (2002). Rebuilding communities, shaping identities: The impact of a participatory neighborhood planning process on young, low-income adolescents of color in $x$, Massachusetts. Unpublished dissertation. University of Massachusetts, Boston.

Ross, L., \& Coleman, M. (2000). Urban community action planning inspires teenagers to transform their community and their identity. Journal of Community Practice. 7, 29-45.

Ross, L., Downs, T., Tejani, A., Dezan, R., \& Lowe, K. (2010). Trust, preparation, transparency, and reflection: Negotiating roles in youth-adult partnerships for social and environmental justice. In W. Linds, L. Goulet, \& A. Sammel (Eds.), Emancipatory practices: Adult/youth engagement for social and environmental justice (pp. 183-198). Rotterdam, The Netherlands: Sense Publishers.

Wong, N., Zimmerman, M., \& Parker, E. (2010). A typology of youth participation and empowerment for child and adolescent health promotion. American Journal of Community Psychology, 46(1/2), 100-114.

Zeldin, S. (2004). Youth as agents of adult and community development: Mapping the process and outcomes of youth engaged in organizational governance. Applied Developmental Science, 8(2), 75-90.

Zeldin, S., Petrokubi, J., \& MacNeil, C. (2008). Youth-adult partnerships in decision making: Disseminating and implementing an innovative idea into established organizations and communities. American Journal of Community Psychology, 41(3/4), 262-277. 